\title{
Engineering a Multi Epitope Vaccine Against SARS- CoV-2 By Exploiting Its Non Structural and Structural Proteins
}

\section{VIKRANT SINGH RAJPUT}

JAWAHARLAL NEHRU UNIVERSITY DELHI INDIA

\section{RITIKA SHARMA}

JAWAHARLAL NEHRU UNIVERSITY DELHI INDIA

ANCHALA KUMARI

JAWAHARLAL NEHRU UNIVERSITY DELHI INDIA

NIDHI VYAS

JAWAHARLAL NEHRU UNIVERSITY DELHI INDIA

VIJAY PRAJAPATI

CENTRAL UNIVERSITY OF RAJASTHAN, RAJASTHAN INDIA

ABHINAV GROVER ( $\square$ agrover@jnu.ac.in )

JAWAHARLAL NEHRU UNIVERSITY DELHI INDIA

\section{Research Article}

Keywords: SARS-COV-2; Immunoinformatics; Multi-epitope; Vaccine; Docking, MD simulation.

Posted Date: July 2nd, 2020

DOl: https://doi.org/10.21203/rs.3.rs-39285/v1

License: (1) This work is licensed under a Creative Commons Attribution 4.0 International License.

Read Full License

Version of Record: A version of this preprint was published at Journal of Biomolecular Structure and Dynamics on July 2nd, 2020. See the published version at https://doi.org/10.1080/07391102.2021.1924265. 


\section{Abstract}

SARS-CoV-2, the causative agent behind the ongoing pandemic exhibits an enhanced potential for infection when compared to its related family members- the SARS-CoV and MERS-CoV; which have caused similar disease outbreaks in the past. The severity of the global health burden, increasing mortality rate and the emergent economic crisis urgently demands the development of next- generation vaccines. Amongst such emergent next generation vaccines are the multi-epitope subunit vaccines, which hold promise in combating deadly pathogens. In this study we have exploited immunoinformatics applications to delineate a vaccine candidate possessing multiple B and T cells epitopes utilizing the SARS-CoV-2 non structural and structural proteins. The antigenicity potential, safety, structural stability and the production feasibility of the designed construct was evaluated computationally. Furthermore, due to the known crucial interactions of human TLR-3 immune receptor with SARS-CoV, the vaccine construct was examined for its binding efficiency using molecular docking and molecular dynamics simulation studies, which resulted in strong and stable interactions. Finally, the immune simulation studies suggested an effective immune response on vaccine administration. Overall, the immunoinformatics analysis advocates that the purposed vaccine candidate is safe and immunogenic and therefore can be pushed as a lead for in vitro and in vivo investigations.

\section{Introduction}

Coronavirus disease (COVID-19) effectuated by the severe acute respiratory syndrome coronavirus 2 virus (SARS-CoV-2), is a globally asserted pandemic by the World Health Organization (WHO $)^{1,2}$. The outbreak was first documented from Wuhan, China, in $2019^{3}$. The highly infectious and deadly virus has been successful in proliferating to 213 countries and territories around the globe till date. According to World Health Organization (WHO), by May 17, 2020, there are 8,043,487 known cases of Covid- 19 worldwide, out of which 3,899,859 cases alone are attributed to America, while Europe has 2,434,184 cases, leading to a death toll of over 4,39,487 worldwide. Coronaviruses belongs to the family Coronaviridae (that encompasses two subfamilies of Coronavirinae and Torovirinae), of the order Nidovirales ${ }^{4,5}$. These are large, enveloped, positive-stranded RNA viruses, with a genome sizet ypically ranging from 27 to $32 \mathrm{~kb}$, largest amongst all RNA viruses. The nucleocapsid protein $(\mathrm{N})$ forms a helical capsid structure that captures the viral genome inside it, this capsid is enclosed by an envelope comprising the membrane (M) protein, envelope (E) protein, and the spike (S) protein ${ }^{6,7}$.

Despite huge efforts by researchers across the world, an effective vaccine or a therapeutic against the menace has not been discovered so far; thereby instigating the expeditious transmission of the virus which is affecting the human populace globally. Lack of adequate knowledge regarding the immune system's response against the viral infection is one of the significant hurdles in the path of SARS-CoV-2 vaccine development. Therefore, vaccine development efforts need to be accelerated using novel 
effective strategies. At the moment, several vaccine design efforts are underway to address this challenge, with considerable advancements in our understanding of the virus's biology 8,9,10,11.

In the past vaccine development against the outbreaks of SARS and MERS have accomplished limited success, which indicates that developing the vaccine against COVID-19 could be a formidable task to achieve ${ }^{11,12}$. Recently, among other approaches, the multi epitope subunit vaccine (MESV) has proved to be an efficacious approach against the virus. Various in silico tools could rationally provide a competent vaccine construct that can be pursued further. A successful vaccine candidate, demands attributes such as high immunogenicity, antigenicity and acceptable physicochemical properties along with no allergenicity and toxicity. The designing of a vaccine using the MESV approach could exactly address these issues ${ }^{13}$. To date numerous vaccines have been entrenched using in silico methods against various viruses such as the Hepatitis $C$ virus, Human immunodeficiency virus- 1 , and many more ${ }^{14,15}$. Recently, Ojha et. al. and Saha and co-workers have utilized the same strategy, to successfully propose a vaccine candidate against the SARS-Cov- ${ }^{9,16}$. Short stretches of amino acid residues from antigenic proteins called as epitopes, are recognisable by B-cell Lymphocytes (BCL), Helper T Lymphocytes (HTL) and Cytotoxic T Lymphocyte (CTL);and may evoke a direct and vigorous immune reaction as compared to the response produced by a whole protein ${ }^{17}$. The availability of the whole genome sequence of SARSCov-2 has provided a better understanding, for the development of vaccines, diagnostics and therapeutics against the same ${ }^{12,9}$.

The development of MESV involves the identification of virulent proteins and thereafter, the selection of non-toxic peptides that can successfully elicit a humoral as well as cellular immune response. Keeping this in view, we engineered the vaccine construct utilizing non-toxic antigenic epitopes against $B(B C L)$ and $T$ cells $\left(H T L / T_{H}\right.$ and $\left.C T L / T_{C}\right)$ by utilizing non-structural proteins viz. non-structural protein 2 (NSP2), non-structural protein 8 (NSP8), and helicase, which are referred as the tool proteins executing the viral replication ${ }^{11}$ and structural proteins, namely nucleoprotein $(\mathrm{N})$ and receptor binding domain (RBD) of the spike (S) protein. NSP2 is known to be conserved in both SARS-CoV and SARS-CoV-2;it bindsto two host proteins, namely prohibitin 1 and prohibitin 2 which are vital for cell cycle passage, cellular differentiation, apoptosis, cell migration and mitochondrial biogenesis. NSP2 binding disrupts the host cellular environment, the major cause of COVID-19 ${ }^{18}$. Further, recent evidences have shown that the highly contagious nature of SARS-Cov-2 might be because of a stabilizing mutation in the endosome associated protein like domain, of the NSP2 protein ${ }^{19}$; thereby making it an important target for vaccine development. NSP8, a peptide cofactor forms a heterodimer with another peptide cofactor, NSP7 and this complex in turn binds NSP12. Along with this complex, an NSP8 monomer also teams up with NSP12, which eventually organizes the RNA polymerase complex ${ }^{18}$. This makes it a valuable protein for vaccine search. The nucleoprotein is a major SARS-CoV structural protein which is known to bind RNA and stabilize it ${ }^{18}$. The SARS-CoV-2 nucleoprotein displays $94 \%$ homology to the nucleoprotein of the SARSCoV; and likewise, it also suppresses RNAi by sequestrating dsRNA; and is thus directly involved in viral RNA replication. Because of this suppression, the $\mathrm{N}$ protein represents an important factor in host 
immune evasion by SARS-Cov-2 and therefore, contributes to its pathogenicity; implying that it can also play a crucial role in developing an effective vaccine ${ }^{20}$. The spike protein (S), facilitates the viral attachment and, thus, the virus entry into the host cells ${ }^{21}$. The RBD of the spike protein specifically recognizes the human Ace2 receptor. This receptor recognition is crucial for the pathogenesis and host range along with viral infectivity, making it another vital target for vaccine development ${ }^{21}$.

Therefore, the above mentioned non structural and structural proteins were utilized to excavate antigenic epitopes that were further combined to generate an antigenic vaccine construct which is non allergenic and non toxic with acceptable physicochemical properties. The vaccine construct was also evaluated for its immunogenic potential, through interaction studies with the human TLR3 immune receptor molecule, via performing molecular docking and Molecular Dynamics (MD) simulation analyses. This was followed by conducting immune-simulation studies that provided an overview of the possible immune response that could be elicited on administration of the purported vaccine.

\section{Materials And Methods}

\subsection{Servers and Tools}

All servers and tools utilized in the study are mentioned in their corresponding sections of usage and their links are provided in supplementary table 8.

\subsection{Selection of Proteins}

The available literature for the SARS-CoV-2was surveyed; and based on it we decided to incorporate structural as well as non-structural proteins of its proteome into creation of a multi-epitope subunit vaccine candidate. The complete peptide sequence for each of the selected proteins was downloaded from the NCBI database, in the form of FASTA files. To ensure that the proteins selected by us exhibited no similarity to any human protein, a BLASTp search was conducted for each individual protein against the human protein dataset. Furthermore, to assess whether the selected proteins had an antigenic capability, the ANTIGENpro tool of the Scratch protein properties interpreter, server was utilized ${ }^{11}$.

\subsection{Identification of B cell epitopes}

The antigenic proteins of SARS-CoV-2, bearing insignificant homology with the human proteome, obtained from the previous step, were individually subjected to analysis by ABCpred server. The ABCpred server is routinely employed in reverse vaccinology for machine learning and artificial neural network (ANN) based computation of amino acid stretches within antigens that can mediate a humoral immune response on interactions with B-lymphocytes under physiological conditions. The default settings of various parameters such as threshold and window size were used in this study ${ }^{11}$.

\subsection{Identification of $T_{H}$ cell epitopes}


For predicting Helper T lymphocytes- MHCII recognised epitopes, the IEDB database was utilized. Individually, each antigenic protein sequence was provided as input to T cell epitope- MHCll binding tool of IEDB, while selecting the complete human HLA reference set in the parameter window. The rationale behind selecting the entire HLA reference setwas that this would yield epitopes covering all the globally present MHC II alleles ${ }^{11}$.

\subsection{Identification of $T_{C}$ cell epitopes}

The prediction of Cytotoxic T Lymphocytes-MHCl binding epitopes was performed by employing the NetCTL 1.2 Server. It utilizes ANN, and yields the immunogenic epitopes, by envisaging functions such as$\mathrm{MHCl}$ binding affinity, proficiency of proteasomal cleavage sites and transporter associated with antigen processing (TAP). The amino acid sequence corresponding to each antigenic protein was provided as input to the server individually. The epitopes were predicted by selecting default parameters for the three $\mathrm{MHCl}$ supertypes- $\mathrm{A} 2, \mathrm{~A} 3$ and $\mathrm{B} 7$, as these three collectively cover $>88 \%$ of the global population ${ }^{11,22,23}$.

\subsection{Assessment of toxicity, prediction of antigenic potency and merging of epitopes to generate the vaccine construct and its efficacy determination}

Toxicity of all the generated epitopes was assessed by employing the ToxinPred server which utilizes models formulated on the technique of machine learning and quantitative matrix, by applying various peptide properties, for predicting toxicity ofthe input peptide/protein sequence ${ }^{11,24}$. Before combining the different non toxic epitopes for fabricating the vaccine construct, the antigenic potential of each of these predicted $B, T_{H}$ and $T_{C}$ cell epitopes were determined by using VaxiJen (version 2.0) server that is available online. VaxiJen, replaces conventional alignment-based strategies with the auto-cross covariance (ACC) methodology to yield reliable results, when predicting antigenicity. A higher VaxiJen score is indicative of an enhanced antigenicity.

The predicted epitopes that yielded lower VaxiJen scores (below the threshold value of 0.4) were left out of the vaccine design study. The remaining epitopes were joined together with the aid of well-known linker/spacer amino acids sequences. The merging of epitopes was performed in the following order starting at the amino $(\mathrm{N})$ terminal, the peptide sequence corresponding to the defensin-3 adjuvant was incorporated; it was linked to the collection of different cell epitopes using the linker sequence 'EAAK'. The $B$ cell epitopes were fused with each other using the 'KK' amino acids, the 'GPGPG' linker was used for combining the different $T_{H}$ cell and the $T_{C}$ cell epitopes were linked in series by means of the ' $A A Y$ ' linker. The different linkers utilized, have been chosen based on previous reports and are known to perform specific functions; such as, increased proteasome processing (KK linker) ${ }^{25}$, improved protein flexibility (GPGPG linker) ${ }^{26}$, site of proteasomal cleavage (AAY linker) ${ }^{27}$, and promoting a helix secondary structure formations (EAAAK linker) ${ }^{11}$ in the designed protein (vaccine molecule). The human beta defensin- 3 is a well-known TLR-3 agonist, and increases the immunogenic potential of a vaccine by enhancing the effectivity of the humoral and cellular immune response; which defines its application in the current study 
${ }^{28,29}$.The VaxiJen server and ANTIGENpro tool of SCRATCH suite were utilized to check the efficacy of the fabricated construct.

\subsection{Physiochemicalproperties and safety assessment of the designed Vaccine construct}

The ExPASy portal's ProtParam tool,and the PepCalc tools were utilized for physiochemical evaluation of the designed vaccine construct ${ }^{11,24}$. Additionally, the possible solubility of this protein upon overexpression in E. coli,was computed by the SOLpro tool of the Scratch suite. For assessing the safety and allergenicity of the proposed vaccine construct, Algpred, AllerTop and AllergenFP web servers were utilized; all three of them are freely available online ${ }^{16,30}$.These servers use sequence matching and machine learning algorithms, for predicting the allergenicity of a given peptide or protein sequence.

\subsection{Prediction of trans-membrane helices}

The membrane localized proteins bearing single or multiple trans-membrane domains, may not be explored by B cells for an immune response. Therefore, the vaccine construct was evaluated for the presence of a possible trans-membrane helix conformation using the TMHMM, TMPred and the TOPCONS servers. The TOPCONS server also evaluates the presence of a possible signal peptide (SigP) cleavage site in the construct ${ }^{31}$.

\subsection{MHC alleles cluster analysis}

The human MHC genomic region (HLA) is known to contain several thousand alleles, and is therefore extremely polymorphic ${ }^{32}$. Thus, it is important to identify MHC allele molecules that might possess similar binding specificities for the vaccine construct. This was accomplished through the MHCcluster server. During the execution, the default values for the parameters which comprise- the sum of peptides to be involved, the number of bootstrap calculations, fraction of peptides to include in correlation analysis were selected; utilizing all the HLA supertypes (MHC class-I) and HLA-DR (MHC class-II) deputies. The server produces a MHC specificity tree and a MHC specificity heat map.

\subsection{Tertiary Structure Prediction}

The three-dimensional structure of the designed multi-epitope vaccine was computed by the 3Dpro tool of the Scratch suite.3Dprocombinesthe use of predicted secondary structural features, a fragment library and energy terms derived from the PDB statistics to generate the tertiary structure of a given amino acid sequence $^{33}$.

\subsection{Modelled Structure's Refinement and Validation}

The predicted 3D models for a given protein sequence seldom have biophysical and topological errors such as unusual bond angles and lengths, irregular contacts or hydrogen bonds, and non-physical atomic clashes; restraining their usage for further studies. Thus, it is essential to alleviate the errors and improve their stereochemistry through structural relaxation. As a single-long model-sampling protocol is usually 
found trapped in local energy minima $34,35,36$, the energetic landscape of constructed model is traversed through Modeller $9.24{ }^{37}$ to select the lowest normalized energy model with the lowest Molprobity score ${ }^{38}$. The sampling is iteratively implemented until convergence ${ }^{39}$. Modeller 9.24 was thus utilized to generate an energy refined structure for the designed vaccine construct. The refined structure was subsequently analysed for structural stability, through online available applications-ProSA-web, PROCHECK and ERRAT.

\subsection{Molecular Interaction Study with TLR-3}

The possibility of an interaction with the immune receptor molecule -TLR3, for the designed vaccine construct was predicted via performing a molecular docking study. The human TLR-3 protein structure (PDB ID: 2AOZ) was downloaded from the RCSB PDB database and the refined vaccine structure obtained as described previously, served as the ligand for this receptor. The interaction between the two was performed using the High Ambiguity Driven protein-protein docking (HADDOCK) server. HADDOCK is different from other docking methods as it integrates a wide variety of experimental and bioinformatics data to guide the modelling process ${ }^{40}$. The active and passive amino acid residues of the receptor as well as the ligand, and their energy minimized PDB structures, are pre-requisites for performing docking by HADDOCK. To obtain these interactive residues, initially the CPROT tool was utilized; CPROT also demands PDB structures of the protein molecules as input. Docking was executed by the HADDOCK server after successful submission of all the input requirements.

\subsection{Molecular dynamics simulations of TLR-3 and premeditated vaccine candidate}

In this study, the TLR-3 and designed vaccine candidate docked complex was simulated with the help of GROMACS (GROningen Machine for Chemical Simulation) v5.0 program, utilizing the GROM0S96 54a7 force field; having water model SPC216 along with time step $1 \mathrm{fs}$ for $20 \mathrm{~ns}{ }^{41}$. It is a command line based application where various trajectory files are generated through all time frames and the motion of every atomic coordinate is defined. The simulation box for the MD run was produced, having a size of $10.075 \mathrm{x}$ $8.901 \times 7.011 \mathrm{~nm}$, which was later filled with about 78659 water molecules of SPC model for TLR-3vaccine candidate complex simulation system. The total charge on TLR-3-vaccine candidate complex was found to be -6.000 e, therefore to neutralize charge +6.000 e were appended into the simulation system by compensating the water molecules in arbitrary locations inside the simulation box. NPT ensembles, along with periodic boundary conditions, were utilized to carry out MD simulations. A cut-off of about $12 \AA$ was used in order to achieve the Vander Waals forces. The Particle Mesh Ewald model having a cut-off of $14 \AA$ was further utilized to compute the electrostatic interactions ${ }^{42}$. The TLR-3vaccine candidate was solvated through a slab of about $10 \AA$ in all directions. The neighbour list was rationalised to a frequency of $10 \mathrm{ps}$.

The simulation system consisted of four key steps. First phase involved the energy minimization of the whole system employing the integrator of steepest descent in perpetuation with subsequent integrator of conjugate gradients algorithms. Second phase comprised of the minimization and molecular dynamics 
of NVT and NPT ensembles for 500 ps and 1000 ps, respectively permitting the solvents and ions to evolve; here the starting configuration for the structures was kept similar. Third phase involved heating of the systems, having a lower temperature coupling $(\tau=0.1 \mathrm{ps})$ along with pressure coupling $(\tau=0.5 \mathrm{ps})$ to achieve equilibrium at $300 \mathrm{~K}$ and $1 \mathrm{~atm}$ of temperature and pressure. In the equilibration phase, the thermostat and barostat were estimated through the Berendsen algorithm ${ }^{43}$. The hydrogen-containing bond lengths were repressed with the help of the LINCS algorithm ${ }^{44}$. The last and fourth phase,termed as the production step is where the MD simulations for $20 \mathrm{~ns}$ at $300 \mathrm{~K}$ temperature having $2 \mathrm{fs}$ of time step were achieved, and the final structures were attained. The Maxwell Boltzmann distribution was employed in order to reassign the velocities at each step. Nose Hoover thermostat and Parrinello Rahman barostat were the respective thermostat and barostat for the final MD or production run ${ }^{43}$.

Various interpretations were achieved with the assistance of inbuilt investigation commands of GROMACS. Root mean square deviation (RMSD) is a magnitude of the dimensional inequality between the two stagnant structures, and RMSD calculation is attained depending upon the native structure and every consecutive trajectory frame in the simulation. Furthermore, root mean square fluctuation (RMSF) profile measures the affability of every protein residue reliant on the fluctuation about an average position within all MD simulations ${ }^{45}$. Therefore, RMSD and RMSF of TLR-3-vaccine candidate complex were determined to scrutinize stability and residual fluctuations. Further, the radius of gyration $\left(R_{g}\right)$ was examined to estimate the compactness of the simulation system. Also, the hydrogen bond study was accomplished to check the neighboring interactions within the simulation system, including the hydrophobic interactions with the help of the DimPlot tool for TLR-3-vaccine complex before and after simulation.

Additionally, solvent accessibility surface area (SASA) was also computed to scrutinize the solvent attributable areas of TLR-3-vaccine candidate complex. Cluster exploration having a cut-off value of 0.25 $\mathrm{nm}$ liable upon the RMSD profile was employed to identify the most prominent conformations, found intermittently throughout the trajectory. Here, all the structures having RMSD values of below $0.25 \mathrm{~nm}$ for all components within a cluster are incorporated to the initial cluster. It is rare that a molecule displaying a RMSD value higher than $0.25 \mathrm{~nm}$ from other cluster, prospectively be treated as a structure. The secondary structure interpretation was also achieved by the DSSP program ${ }^{46}$. The visualization of protein nature throughout the entire simulation was accomplished through Visual Molecular Dynamics (VMD) ${ }^{47}$ and UCSF Chimera ${ }^{48}$.

\subsection{Immune simulation}

The in silico immune reaction produced by the proposed vaccine construct was determined using CImmSim server ${ }^{49}$. It is an online available, dynamic immune simulation tool that produces well constructed and compatible results, by utilizing basic principles of immunology. The multi-level model expresses the immune reaction at the mesoscopic level, while considering the perception operations between immune system mediators, based on prediction tools for epitope identification. The default 
parameters were selected while providing the amino acid sequence of the proposed vaccine candidate as an input for executing the study.

\subsection{In silico cloning}

For cloning and over-expressing the proposed vaccine candidate in an appropriate $E$. coli expression vector, the protein sequence of the construct was reverse translated into the corresponding coding DNA sequence by utilizing the Gene infinity server, assembled on the codon usage of the E.coli K12 strain. Thereafter, using the GenScript tool, the optimized DNA sequence was examined; based on parameters that are crucial for successful recombinant protein expression. These parameters include GC content, codon adaptation index (CAI), and codon frequency distribution (CFD ${ }^{24}$. Finally, in silico cloning was executed through the WebDSV ver. 2.0 server using the sequence of the expression vector, pET28a (+) which was retrieved from the 'addgene' vector database ${ }^{50}$.

\section{Results}

\subsection{Selection of antigenic proteins, their retrieval and homology with human proteome}

There exist two kinds of proteins in SARS-Cov-2 proteome, the non structural and the structural proteins. The non structural proteins principally participate in the process of viral replication, whereas the structural proteins function in the progression of viral assembly and infection within the host ${ }^{51}$. Therefore, both classes of proteins should bear equal consideration while either developing a vaccine or a drug. In this study too, we considered a combination of both non structural and structural proteins for fabricating a vaccine. These proteins included non structural 2 (NSP2), non structural 8 (NSP8), helicase (H), Nucleocapsid protein $(\mathrm{N})$ and receptor binding domain (RBD) of spike (S) protein. All of them were retrieved from NCBI database and evaluated for homology against the human proteome using a BLASTp search. The viral helicase displayed $22.4 \%$ of sequence identity to the human ZGRF1 isoform X10 protein; with a query coverage of just $34 \%$. Since, the probability of cross reactivity is considered as very low, if the similarity between human and pathogen proteins is less than $40 \%{ }^{52}$ we were able to select helicase for our further studies. Similarly, the nucleoprotein, exhibited $55.56 \%$ identity to the human immunoglobin heavy chain junction region which although appears high, but considering the exhibited query coverage value of just $4 \%$,and a high e-value of 5.4 in the BLASTp results, was deemed insignificant. On this basis nucleocapsid protein was also taken forward into the investigation. All the other proteins did not show any similarity against the human proteome. Further, these proteins were evaluated for their antigenic potential using the ANTIGENpro tool of the Scratch protein properties predictor server. All the proteins were found to be highly antigenic and therefore, were subsequently examined for epitope generation and vaccine designing (Supplementary Table 1).

\subsection{Identification of B cell epitopes}

The ABCPred server predicted the B cell epitopes against each selected protein. Based on their ranking and the highest score, the best epitope of 10 mer length for each protein was chosen for further 
investigations (Supplementary Table 2).

\subsection{Identification of $T_{H}$ cell epitopes}

The IEDB database was utilized to predict Helper T lymphocytes- MHCll recognised epitopes for each protein. The percentile rank andlC $\mathrm{C}_{50}$ values were the main criteria utilized for selection of the predicted epitopes (specifically a cut off in percentile rank of less than or equal to 0.9 and anI $\mathrm{C}_{50}$ value of below or

equal 50). Lower the percentile rank and the $\mathrm{IC}_{50}$ value, better is the binding efficiency of the epitope ${ }^{11}$. In order to cover maximum population possible, the complete human HLA reference set was stipulated in the parameter window. The best two epitopes for each protein were selected. The preferred $T_{H}$ cell epitopes with their $\mathrm{IC}_{50}$ value and the percentile rank score are presented in supplementary file in table 3 .

\subsection{Identification of $T_{C}$ cell epitopes}

The Net-CTL server was applied to identify the $T_{C}$ cell epitopes. Barring the supertype selection, all other parameters such as threshold value (0.75), C-terminal cleavage (0.15), and TAP transport efficiency (0.05) were set to default values. The HLA supertype selection determines the specific and efficient perception of epitopes for T cell's, aimed at several viral diseases, including SARS, MERS, HIV and MMR ${ }^{11}$. Based on the fact that A2, A3 and B7 HLA supertypes together cover more than $88 \%$ of the global population, epitopes were predicted by selecting these supertypes in the parameter window. The study resulted in identification of 15 epitopes, 3 each from every protein (Supplementary Table 4).

\subsection{Prediction of antigenicity of the epitopes, assessment of toxicity and construction of the vaccine construct and its efficacy determination}

All the epitopes were evaluated for their antigenicity using the VaxiJen server (Supplementary Table 2, 3 and 4). Those epitopes which exhibited VaxiJen scores below the threshold value of 0.4 , were left out from further studies. In addition, the plausible toxicity of all the B and T cell epitopes was examined using the ToxinPred server; and all of them were predicted to be safe and non toxic (Supplementary Table 5, 6 and 7). The non toxic, $B, T_{H}$ and $T_{C}$ cell epitopes which exhibited VaxiJen scores higher than the threshold were used to create the vaccine construct.

The selected epitopes (antigenic peptides) were coupled together, utilizing the linkers to yield the vaccine candidate. These linkers, not only assist in maintenance of construct immunogenicity, but also in making the resulting protein complex more flexible and stable. ${ }^{11}$ The B cell epitopes were linked using linkers 'KK' while the $T_{H}$ cell and the $T_{C}$ cell epitopes were connected through 'GPGPG' and 'AAY' linkers, respectively. Finally, the 'EAAAK' linker joined the adjuvant - human beta defensin-3 (which is known to augment a sturdy $B$ and $T$ cell response ${ }^{29,28}$ ) to the assembled epitopes for generating the vaccine construct (Supplementary Figure 1). On evaluating the generated vaccine construct for its antigenicity, score values of 0.6033 and 0.836364 were obtained by VaxiJen and ANTIGENpro respectively, confirming the strong immunogenic potential of the proposed multi subunit vaccine. 


\subsection{Physiochemical properties and safety assessment of the designed Vaccine construct}

Various physicochemical properties were determined for the vaccine construct using the ProtParam and PepCalc A tools. Both the tools yielded acceptable physicochemical properties for the vaccine construct. Briefly, the instability index was found to be 20.26 (should be below 40); half-life of the protein was predicted to be approximately- 30 hours in mammalian reticulocytes, $>20$ hours in yeast and $>10$ hours in E. coli. The calculated GRAVY (Grand average of hydropathicity) score was -0.273 ; the value of aliphatic index of the protein was 77.4 , the instability index (II) was determined to be 20.26 , and the construct was predicted to display good water solubility. Additionally, solubility upon recombinant expression was examined using the SOLpro tool, where the protein was anticipated to be soluble with a probability score of 0.793223 . Table 1 depicts all these properties, evaluated by the mentioned tools.

The AlgPred server predicted the possible allergenicity for the vaccine construct. It applies six methods (IgE mapping, MEME/Mast motif, amino acid as well as dipeptide composition based SVM modules, BLAST inspection on ARPs and a hybrid approach that encompasses all these parameters) for determination of protein allergenicity. Among these methods, the SVM modules that take amino acid and dipeptide composition into consideration predicted the protein as allergenic; whereas the hybrid approach, MEME/Mast motif, BLAST search on ARPs andlgE surveying predictors classified the vaccine construct as non-allergenic. In addition, evaluation of the construct using the AllerTop and AllergenFP servers also identified the protein to be non-allergenic. Considering these results, the proposed vaccine candidate can be indexed as a probable non allergen.

\subsection{Prediction of the trans-membrane helix}

The trans-membrane helix forming regions, or the membrane spanning peptide portion of a protein is immunologically non-accessible. The vaccine construct has no noteworthy alpha-helix trans-membrane (TM) topology as concluded by the TMHMM probability plot (Supplementary Figure 2A). Using the TMPred web-server, scores greater than 500 are considered as crucial for TM helix prediction. The proposed vaccine construct exhibited three fragments, 89-110 (score 633) in inside-to-outside (i-o) orientation, 130-149 (score 562) inoutside-to-inside (o-i) orientation and 283-301 (score 1064) inside-tooutside (i-o) orientation when computed through the strongly preferred model (Supplementary Figure 2 B). Whereas the alternative model predicted two TM helices, viz. $92-110$ (score 1136) in outside-to-inside (o-i) orientation and 283-301 (score 1064) in inside-to-outside (i-o) orientation (Supplementary Figure 2B). The TOPCONS server which utilizes six algorithms to detect TM helix was also applied. Neither the TM helix nor a signal sequence was predicted in the vaccine construct by this server (Supplementary Figure 2C).

\subsection{MHC cluster analysis}

The potential $\mathrm{MHC}$ I and $\mathrm{MHC}$ II allele interactions with the epitopes in the vaccine candidate were examined by the MHCcluster server. The relationship of the cluster of alleles with the epitopes is 
represented in the form of a specificity heat map and tree (Supplementary Figure 3). The red and the yellow zone symbolize strong and weaker interactions respectively.

\subsection{Tertiary structure prediction and quality assurance of the designed candidate vaccine}

The 3Dpro module of Scratch protein predictor, web-server estimated the likely 3Dconfigurationof the proposed vaccine candidate. On analysis by structure visualization tools such as PyMol and UCSF Chimera, it was observed that the designed multi-epitope vaccine majorly comprised of alpha helices, with very little contribution from a beta sheet structure. Structural refinement was performed by Modeller 9.24 and the refined structure (Figure 1A) was validated using ProSA, PROCHECK and ERRAT2.ProSA estimates a global quality score for the structure provided as input; the structure is considered free of errors if the ProSA-z score falls within a limit that is representative of native proteins having similar number of residues. The ProSA score for the fabricated vaccine construct's structure was observed to be -5.38 and as is visible from the figure $1 B$ and $C$, it falls well within the range, and thus is confirmed for its quality. The PROCHECK generated Ramachandran plot validated the quality of the 3D structure, as most $96 \%$ of the residues were positioned in the most favoured regions and only $0.4 \%$ of them were localized in the disallowed regions (Figure 1D). Analysis by ERRAT2 predicted the overall quality factor as 93.233 (signified as proportion of the protein for which the computed rate of structural inaccuracy lies within the 95\% elimination limit) (Figure 1E).

\subsection{Molecular Docking of the Vaccine construct with TLR-3}

For activation of an immune response a suitable interaction amidst the immune receptor and the antigen molecules is essential. Therefore, to assure that the designed vaccine candidate is capable of associating with TLR-3 we performed a docking study using HADDOCK web-server. HADDOCK clustered 40 structures of the docked complex in a total of 6 clusters, the cluster with the best statistics (RMSD values; Van der Waals, Electrostatic and Desolvation energy values) was found to have a Z score of -1.9; lowest of all the clusters. The more negative the $\mathrm{Z}$ score, the better is the interplay between the receptor and the ligand. The details of the topmost docked cluster are given in the table 2 and the binding of the vaccine candidate to TLR-3 is represented in figure 2.

\subsection{Simulation}

MD simulation for the docked complex of TLR-3 and the designed vaccine was evaluated through trajectory study. For the progression of 20 ns MD run, the stable trajectory was perceived and the representative structures were acquired. Different steps for energy minimization, pressure and density equilibrium, and temperature persistence were analysed (Supplementary Figure 4). The deviation of the backbone atoms for simulated structures, comparative to the starting structures, was utilized as a reference and was assessed through RMSD (Figure 3A). Steep RMSD variation during the entire simulation can be an insinuation of a malleable and free instinctive protein or the variation of the force field. Depending upon the effects of the RMSD assessment, Figure 3A signifies that the RMSD fluctuation stabilizes, around 10 ns into the MD simulation; and thus, the total time for simulation was adequate. In 
the time frame 10-20 ns, RMSD for TLR-3-vaccine candidate complex has an approximate value of about $0.7-0.8 \mathrm{~nm}$.

The fundamental magnitude of this principle is attained by evaluating the disparities arising from modifications of each of the protein residues that majorly feature the most flexible chain frames. Hence, we validated the residual fluctuations by calculating the mean fluctuations for the stable trajectory of the simulation. The RMSF evaluation of all protein residues was attained in order to check the residues that may have inclined to an enhancement in the RMSD results (Figure 3B). Convincing fluctuations existed in the terminal residues, few $\beta$-sheet regions along with the loops linking the alternative $\beta$-sheets of TLR-3 (residues 330-350, and 490-550) to about $0.32 \mathrm{~nm}$, and few a-helical sections of vaccine candidate (residues 130-210) to about $0.73 \mathrm{~nm}$. Also, we noticed that residues 299-355 of TLR-3 showed comparatively reduced discrepancy in RMSF values; these residues form the binding segment for the corresponding vaccine candidate residues (175-272) after MD simulation. Interestingly, the binding region for TLR-3 and the designed vaccine before and after MD simulation was found to be similar, suggesting a strong binding interaction of the vaccine designed towards TLR-3, and provides stability to the docked complex (Figure 4A).

The radius of gyration investigation was achieved to determine the modification in compactness of TLR3 and designed vaccine throughout the MD run. The $R_{g}$ plot shows decrease in radius of gyration values and hence increase in compactness of the protein complex, persists after MD simulation (Figure 3C). The compactness of the TLR-3 protein complex is due to the strong binding interaction of the designed vaccine. Similar observations were determined through SASA analysis representing the solvent defined protein surface and its orientation through folding, making the alterations in the exposed and buried regions of the surface area of proteins. SASA values for the simulation was about $485 \mathrm{~nm} / \mathrm{S}^{2} / \mathrm{N}$ after 15 ns (Figure 3D). Also, it remains similar till 20 ns suggesting that TLR-3-vaccine solvation profile shows a convincing SASA value suggesting a stable structure and strong binding interaction with the vaccine candidate.

Also, the hydrogen bond landscape was evaluated, which exposed the dynamic equilibration of the complex trajectory with a high number of hydrogen bonds, as shown in Figure 5. The consistent high numbers of hydrogen bonds were perceived which contributed significantly to the proximal binding of the designed vaccine with the TLR-3 receptor. Further, these results are strengthened by the vital contribution by the complex binding energies throughout the simulation run. These calculations with consistent high binding energies and large hydrogen bonds involvement demonstrate the stable binding of candidate vaccine with TLR-3.

Further, cluster analysis having a RMSD based cut-off value of $0.25 \mathrm{~nm}$ confirmed the development of 17 distinctive clusters for TLR-3 vaccine complex system. The most dominant cluster attained after $10 \mathrm{~ns}$ of MD simulation is depicted in supplementary figure 5 . Also, a secondary structure investigation of the stable trajectory was implemented by the DSSP tool of GROMACS. TLR-3-vaccine complex was formed mainly of continuous coils and conserved $\beta$-sheet regions as secondary structure elements infused with 
various small segments of bend, a-helix, turn, and $\beta$-bridge (Figure 4B). Both cluster analysis and secondary structure interpretation reveals the conformational modifications before and after simulations for TLR-3 vaccine complex structure.

\subsection{Immune simulation}

The C-ImmSim server was utilized for immune simulation study, which gives a picture of the possible immune response on vaccine administration. The peaks for $\lg \mathrm{G} 1+\lg \mathrm{g} 2$ and $\lg \mathrm{M}$ showa secondary and tertiary immune reaction along with the presence of $\lg \mathrm{G}+\operatorname{lgM}$, all of which seems to peak between 10-15 days (Figure 6A). The active $B$ cell population was stimulated, which peaks in around 8 days and remains stagnant for many days (Figure6B). Similarly, the cell mediated immune response which is led by $T_{C} \& T_{H}$ cells was also found to be high (Figure $6 \mathrm{C}$ and $6 \mathrm{D}$ ). The IFN- $\gamma$ level was also found to be inflated during vaccine administration (Figure $6 \mathrm{E}$ ). These results indicate a high probability, of generating a competent immune response which can potentially control the SARS-Cov-2 pandemic.

\subsection{In silico cloning}

The vaccine construct was reverse translated into the corresponding nucleotide sequence and codon optimization was executed using the GenScript tool, which also predicts crucial parameters to forecast protein expression in the host. The CAI value and GC content of the sequence was 1 and $62.37 \%$ respectively, along with $0 \%$ CFD of the gene in E. coli (Supplementary Figure 6). These outcomes imply that the optimized nucleotide sequence is suitable for cloning and expression in $E$. coli ${ }^{53}$. The in silico cloning was performed using the WebDSV 2.0 server, utilizing the of pET28a (+) expression vector's HindIII and BamHI restriction sites. The results of in silico cloning are presented in the figure 7.

\section{Discussion}

The severe acute respiratory syndrome coronavirus 2 (SARS-CoV-2) has led to alarming numbers of fatalities across the world causing a global health emergency. Researchers worldwide have not been successful till date in advancing to a cure, or preventing the disease through a potent drug or an effective vaccine. Therefore, it is imperative to unfold an efficacious vaccine which may contain the ongoing menace. Owing to the exhaustive and extortionate process for assessment of laboratory efficacy of a vaccine, the in silico approaches for vaccine design are gaining interest; and can potentially expedite the vaccine development process, by providing lead constructs that can be pursued further. Computational immunology or immunoinformatics, utilizes computational strategies to investigate different immunological events, along with the development of algorithms for identifying potent $B$ and $T$ cell epitopes. The application of immunoinformatics for designing multi-epitope subunit vaccines, is a cost effective modus operandi against infectious pathogens, displaying high degrees of specificity, safety and stability values ${ }^{10,54,55,56}$. Computational vaccinology technique has proven its value against various infectious diseases. Utilizing the immunoinformatics strategies, many effective vaccines against 
pathogens like Chlamydia pneumoniae, Streptococcus pneumonia ${ }^{57}$, Rickettsia prowazekii ${ }^{58}$, enterotoxigenic E. coli ${ }^{59}$ and Staphylococcus aureus ${ }^{60}$ have emerged.

In the present study, we have exploited immunoinformatics to delineate a novel multi-epitope subunit vaccine against the deadly SARS-Cov-2. For this purpose, five vital proteins displaying significant immunogenicity and no homology to humans were selected. Although helicase displayed $22.4 \%$ sequence identity to the human protein ZGRF1 isoform, but since the chances of cross reactivity are negligible when the sequence similarity is below $40 \%$ between the pathogen and human ${ }^{11}$, helicase was considered for the study. Similarly, the nucleoprotein displayed $55.56 \%$ identity with the human immunoglobin heavy chain junction region, but with a meagre $4 \%$ query coverage value and a high $\mathrm{E}$ value of 5.4, this similarity was considered insignificant. In order to generate an effective humoral and cellular immune response by a vaccine candidate, the epitopes ought to be accessible to $B$ cells as well as $\mathrm{MHC} I$ and $\mathrm{MHC}$ II molecules ${ }^{61}$. Therefore, $\mathrm{B}$ and $\mathrm{T}$ cell epitopes were mapped using online web servers. In order to cover largest achievable population coverage, the full HLA reference set was selected for HTL epitopes identification and similarly; supertypes A2, A3 and B7, which jointly enclose about $88 \%$ population worldwide were chosen for CTL determination ${ }^{11,22,23}$. Based on either ranking or highest score (B cell), lowest percentile rank and $\mathrm{IC}_{50}$ value $(\mathrm{HTL})$ and high score $(\mathrm{CTL})$, the best possible epitopes were selected. These epitopes were once again scrutinized for antigenicity individually, and only those epitopes which displayed significant antigenicity potential were carried forward in the investigation. These epitopes were further studied for their physicochemical properties, allergenicity and toxicity; computationally. Fortunately, none of the selected epitope displayed any of the unfavourable properties. These epitopes were then used to generate the vaccine construct using appropriate linkers. The vaccine construct was also evaluated for its antigenicity along with unfavourable properties if any. The proposed vaccine construct displayed significant antigenicity using two online web servers (VaxiJen and ANTIGENpro), and did not exhibit any unacceptable characteristics. The evaluation of trans-membrane forming regions in the vaccine construct, which represents an inaccessible portion for B cells, showed absence of any such domains. Moreover, MHC class-I alleles and MHC class-II alleles cluster analysis was performed to functionally cluster them in accordance with their predicted binding specificity and to infer their association with each other.

The structure corresponding to the protein sequence of the vaccine candidate was envisaged by 3Dpro, to have majorly an alpha helical conformation, this generated structure was refined to remove biophysical and topological errors via energy minimization through Modeller 9.24. The refined structure so generated was observed to be convincing for further computational experiments. The ProSA Z score was noticed to be within the distinctive range for a native protein. The Ramachandran plot generated through PROCHECK validated the quality of the structure. The modelled vaccine candidate displayed only $0.4 \%$ of the residues in disallowed regions, thereby indicating minimum steric hindrance created among side chain and main chain atoms. Further, the ERRAT server analysis, which identifies the arrangement of nonbonded atomic interactions, was carried out. The ERRAT score (93.233), which is an indicator of overall quality suggests that a high quality model is produced. 
Previous reports suggest that mice in which TLR-3 and TLR-4 are absent, are more prone to SARS-CoV contagion when compared to the mice possessing them ${ }^{62}$. Additionally, it is observed that TLR-3 is mainly involved in recognition of viral ds-RNA; thus, it plays a crucial role during coronavirus infection. Based on these observations, the vaccine construct was evaluated for molecular docking on TLR-3 using the HADDOCK server to showcase the possible interactions. On performing docking, 6 clusters of the docked complexes were obtained. Cluster 1 with the best statistics was observed to have a Z score of -1.9 , the lowest value attained among all the clusters, indicating a high efficiency binding. In sum, the designed vaccine construct/protein was successful in binding with the human TLR-3 immune receptorwith a good docking score. Furthermore, the docking complex of TLR-3 and designed vaccine got stabilized within $~ 10$ ns into the simulation, and the 20 ns simulation trajectory conformation displayed negligible topological variations in the range of $0.7-0.8 \mathrm{~nm}$. The residue network of the interacting substructure of TLR-3 and the designed vaccine was found quite similar during and after the run, and it reliably confirms the topological stability. The increased compactness of the complex indicates a strong binding of TLR-3 and the designed vaccine. The difference between the exposed and buried surface area and a strong hydrogen-bonding network further affirm the significant potential of the designed vaccine.

By carrying out the immune simulation experiment, which mimics the innate framework of an immune system, the immune response from the major stakeholders of the immune system namely, B cells and $T$ cells which are involved in humoral and cellular responses, respectively at different doses and at varied intervals against the SARS-Cov-2 multi-epitope subunit vaccine was affirmed ${ }^{11}$. The outcome from this study indicates a powerful immunereaction by the proposed vaccine construct. Finally, in order to push the proposed vaccine construct towards laboratory evaluation, it is crucial that the optimum-level of protein expression in hostssuch as E. coli could be achieved. Therefore, CAI, CFD, and GC content of the gene are some of the variables that need to be optimized. Our results very well support the proposed vaccine construct gene in this regard ${ }^{24}$.

In sum, the study indicates that the proposed vaccine candidate bears competent structural, acceptable physiochemical and desirable immunological attributes that can elicit a powerful humoral and cellular immune reaction and therefore should be pushed as a prospective lead candidate for further in vitro and in vivo investigations against SARS-CoV-2.

\section{Declarations}

\section{Acknowledgements}

VSR is thankful to Dept. of Health Research (Govt. of India) for the young scientist fellowship (No. 12014/13/2018-HR/E-Office: 3151268). RS and AK are contended to Indian Council of Medical Research (ICMR), India for the SRF position. AG is thankful to University Grant Commission, India for the faculty Recharge position. NV is thankful to DST Inspire fellowship for research grant.

\section{Disclosure statement}


The authors declare no conflicting interests.

\section{Contributions}

VSR conceptualized the whole study. VSR and RS designed and conducted all the studies pertaining to epitope identification and selection. AK carried out the structure generation, in silico docking and MD studies. NV assisted in data curation and manuscript writing. VP guided the study and reviewed the manuscript. AG managed resources and reviewed the manuscript. All authors read and approved the manuscript.

\section{References}

1. Perlman, S. \& Netland, J. Coronaviruses post-SARS: Update on replication and pathogenesis. Nat. Rev. Microbiol. 7, 439-450 (2009).

2. Bianchi, M., Benvenuto, D., Giovanetti, M., Angeletti, S. \& Pascarella, S. Sars-CoV-2 Envelope and Membrane proteins: differences from closely related proteins linked to cross-species transmission? Preprints 2020, 1-14 (2020).

3. Riou, J. \& Althaus, C. L. Pattern of early human-to-human transmission of Wuhan 2019 novel coronavirus (2019-nCoV), December 2019 to January 2020. Eurosurveillance 25, 1-5 (2020).

4. Emmanuel, C. Coronaviruses with SARS-CoV-2 (causative agent for COVID-19) as a case study. 2, (2020).

5. Cavanagh, D. Coronaviridae: a review of coronaviruses and toroviruses. Coronaviruses with Spec. Emphas. First Insights Concern. SARS 1-54 (2005) doi:10.1007/3-7643-7339-3_1.

6. Venkatagopalan, P., Daskalova, S. M., Lopez, L. A., Dolezal, K. A. \& Hogue, B. G. Coronavirus envelope (E) protein remains at the site of assembly. Virology 478, 75-85 (2015).

7. Nieto-Torres, J. L. et al. Subcellular location and topology of severe acute respiratory syndrome coronavirus envelope protein. Virology 415, 69-82 (2011).

8. Ojha, R., Pandey, R. K. \& Prajapati, V. K. Vaccinomics strategy to concoct a promising subunit vaccine for visceral leishmaniasis targeting sandfly and leishmania antigens. Int. J. Biol. Macromol. 156, 548-557 (2020).

9. Ratnadeep Saha, B. V. L. S. P. In silico approach for designing of a multi-epitope based vaccine against novel Coronavirus (SARS-COV-2). bioRxiv 9, 10-16 (2020).

10. Suhrbier, A. Multi-epitope DNA vaccines. Immunol. Cell Biol. 75, 402-408 (1997).

11. Ojha, R. et al. High throughput and comprehensive approach to develop multiepitope vaccine against minacious COVID-19. Eur. J. Pharm. Sci. 151, 105375 (2020).

12. Hilgenfeld, R. \& Peiris, M. From SARS to MERS: 10 years of research on highly pathogenic human coronaviruses. Antiviral Res. 100, 286-295 (2013).

13. Urrutia-Baca, V. H. et al. Immunoinformatics Approach to Design a Novel Epitope-Based Oral Vaccine Against Helicobacter pylori. J. Comput. Biol. 26, 1177-1190 (2019). 
14. Nezafat, N. et al. Designing an efficient multi-epitope peptide vaccine against Vibrio cholerae via combined immunoinformatics and protein interaction based approaches. Comput. Biol. Chem. 62, 82-95 (2016).

15. Yang, Y. et al. In silico design of a DNA-based HIV-1 multi-epitope vaccine for Chinese populations. Hum. Vaccines Immunother. 11, 795-805 (2015).

16. Naik, B. et al. High throughput virtual screening reveals SARS-CoV-2 multi-target binding natural compounds to lead instant therapy for COVID-19 treatment. Int. J. Biol. Macromol. 160, 1-17 (2020).

17. Kao, D. J. \& Hodges, R. S. Advantages of a synthetic peptide immunogen over a protein immunogen in the development of an anti-pilus vaccine for Pseudomonas aeruginosa. Chem. Biol. Drug Des. 74, 33-42 (2009).

18. Yoshimoto, F. K. The Proteins of Severe Acute Respiratory Syndrome Coronavirus-2 (SARS CoV-2 or n-COV19), the Cause of COVID-19. Protein J. 39, 198-216 (2020).

19. Silvia Angeletti, Domenico Benvenuto, Martina Bianchi, Marta Giovanetti \&, Stefano Pascarella, M. C. COVID-2019 The roleofthe nsp2 and nsp3 in its pathogenesis. 584-588 (2020).

20. Mu, J. et al. SARS-CoV-2-encoded nucleocapsid protein acts as a viral suppressor of RNA interference in cells. Sci. China Life Sci. 1-4 (2020) doi:10.1007/s11427-020-1692-1.

21. Shang, J. et al. Structural basis of receptor recognition by SARS-CoV-2. Nature 581, 221-224 (2020).

22. Sette, A. \& Sidney, J. Nine major HLA class I supertypes account for the vast preponderance of HLA-A and -B polymorphism. Immunogenetics 50, 201-212 (1999).

23. Shen, W. J., Zhang, X., Zhang, S., Liu, C. \& Cui, W. The utility of supertype clustering in prediction for class II MHC-peptide binding. Molecules 23, 1-18 (2018).

24. Nosrati, M. et al. Designing a multi-epitope vaccine for cross-protection against Shigella spp: An immunoinformatics and structural vaccinology study. Mol. Immunol. 116, 106-116 (2019).

25. Yano, A. et al. An ingenious design for peptide vaccines. Vaccine 23, 2322-2326 (2005).

26. Livingston, B. et al. A Rational Strategy to Design Multiepitope Immunogens Based on Multiple Th Lymphocyte Epitopes. J. Immunol. 168, 5499-5506 (2020).

27. Ojha, R., Nandani, R. \& Prajapati, V. K. Contriving multiepitope subunit vaccine by exploiting structural and nonstructural viral proteins to prevent Epstein-Barr virus-associated malignancy. J. Cell. Physiol. 234, 6437-6448 (2019).

28. Mohan, T., Sharma, C., Bhat, A. A. \& Rao, D. N. Modulation of HIV peptide antigen specific cellular immune response by synthetic $\alpha$ - and $\beta$-defensin peptides. Vaccine 31, 1707-1716 (2013).

29. Gupta, N. et al. Receptor-ligand based molecular interaction to discover adjuvant for immune cell TLRs to develop next-generation vaccine. Int. J. Biol. Macromol. 152, 535-545 (2020).

30. Dimitrov, I., Naneva, L., Doytchinova, I. \& Bangov, I. AllergenFP: Allergenicity prediction by descriptor fingerprints. Bioinformatics 30, 846-851 (2014).

31. Pourseif, M. M., Yousefpour, M., Aminianfar, M., Moghaddam, G. \& Nematollahi, A. A multi-method and structure-based in silico vaccine designing against Echinococcus granulosus through 
investigating enolase protein. Biolmpacts 9, 131-144 (2019).

32. Thomsen, M., Lundegaard, C., Buus, S., Lund, O. \& Nielsen, M. MHCcluster, a method for functional clustering of MHC molecules. Immunogenetics 65, 655-665 (2013).

33. Cheng, J., Randall, A. Z., Sweredoski, M. J. \& Baldi, P. SCRATCH: A protein structure and structural feature prediction server. Nucleic Acids Res. 33, 72-76 (2005).

34. Runthala, A. Short review: Protein structure prediction: Challenging targets for CASP10. J. Biomol. Struct. Dyn. 30, 607-615 (2012).

35. Runthala, A. \& Chowdhury, S. Refined template selection and combination algorithm significantly improves template-based modeling accuracy. J. Bioinform. Comput. Biol. 17, 1-21 (2019).

36. Garg, S., Kakkar, S. \& Runthala, A. Improved Protein Model Ranking through Topological Assessment. Comput. Biol. Bioinforma. 406-424 (2016) doi:10.1201/b20026-24.

37. Webb, B. \& Sali, A. Comparative protein structure modeling using MODELLER. Curr. Protoc. Bioinforma. 2016, 5.6.1-5.6.37 (2016).

38. Chen, V. B. et al. MolProbity: All-atom structure validation for macromolecular crystallography. Acta Crystallogr. Sect. D Biol. Crystallogr. 66, 12-21 (2010).

39. Runthala, A. \& Chowdhury, S. Iterative optimal TM-score and Z-score guided sampling significantly improves model topology. Lect. Notes Eng. Comput. Sci. 2209, 123-128 (2014).

40. Van Zundert, G. C. P. et al. The HADDOCK2.2 Web Server: User-Friendly Integrative Modeling of Biomolecular Complexes. J. Mol. Biol. 428, 720-725 (2016).

41. Abraham, M. J. et al. Gromacs: High performance molecular simulations through multi-level parallelism from laptops to supercomputers. SoftwareX 1-2, 19-25 (2015).

42. Darden, T., York, D. \& Pedersen, L. Particle mesh Ewald: An N·log(N) method for Ewald sums in large systems. J. Chem. Phys. 98, 10089-10092 (1993).

43. Berendsen, H. J. C., Postma, J. P. M., Van Gunsteren, W. F., Dinola, A. \& Haak, J. R. Molecular dynamics with coupling to an external bath. J. Chem. Phys. 81, 3684-3690 (1984).

44. Hess, B., Bekker, H., Berendsen, H. J. C. \& Fraaije, J. G. E. M. LINCS: A Linear Constraint Solver for molecular simulations. J. Comput. Chem. 18, 1463-1472 (1997).

45. Knapp, B., Frantal, S., Cibena, M., Schreiner, W. \& Bauer, P. Is an intuitive convergence definition of molecular dynamics simulations solely based on the root mean square deviation possible? J. Comput. Biol. 18, 997-1005 (2011).

46. Martin, J. et al. Protein secondary structure assignment revisited: A detailed analysis of different assignment methods. BMC Struct. Biol. 5, 1-17 (2005).

47. William Humphrey, Andrew Dalke, and K. S. VMD: Visual Molecular Dynamics. J. Mol. Graph. 14, 3338 (1996).

48. Pettersen, E. F. et al. UCSF Chimera - A visualization system for exploratory research and analysis. J. Comput. Chem. 25, 1605-1612 (2004). 
49. Rapin, N., Lund, O., Bernaschi, M. \& Castiglione, F. Computational immunology meets bioinformatics: The use of prediction tools for molecular binding in the simulation of the immune system. PLoS One 5, (2010).

50. Bhattacharya, M. et al. Computer aided novel antigenic epitopes selection from the outer membrane protein sequences of Aeromonas hydrophila and its analyses. Infect. Genet. Evol. 82, 104320 (2020).

51. Mirza, M. U. \& Froeyen, M. Structural elucidation of SARS-CoV-2 vital proteins: Computational methods reveal potential drug candidates against main protease, Nsp12 polymerase and Nsp13 helicase. J. Pharm. Anal. (2020) doi:10.1016/j.jpha.2020.04.008.

52. Pearson, W. R. An introduction to sequence similarity ('homology') searching. Curr. Protoc. Bioinforma. 1-8 (2013) doi:10.1002/0471250953.bi0301s42.

53. Mohammadi, S. et al. In silico Analysis of Different Signal Peptides for the Excretory Production of Recombinant NS3-GP96 Fusion Protein in Escherichia coli. Int. J. Pept. Res. Ther. 25, 1279-1290 (2019).

54. Patronov, A., \& Doytchinova, I. (2013). T-cell epitope vaccine design by immunoinformatics. Open Biology, 3(1), 120139. Open Biol. 3, 120139 (2013).

55. De Groot, A. S., Sbai, H., Aubin, C. Saint, McMurry, J. \& Martin, W. Immuno-informatics: Mining genomes for vaccine components. Immunol. Cell Biol. 80, 255-269 (2002).

56. Nosrati, M., Mohabatkar, H. \& Behbahani, M. A Novel Multi-Epitope Vaccine For Cross Protection Against Hepatitis C Virus (HCV): An Immunoinformatics Approach. Res. Mol. Med. 5, 17-26 (2017).

57. Pourhajibagher, M. \& Bahador, A. Designing and in Silico Analysis of PorB Protein from Chlamydia Trachomatis for Developing a Vaccine Candidate. Drug Res. (Stuttg). 66, 479-483 (2016).

58. Caro-Gomez, E., Gazi, M., Goez, Y. \& Valbuena, G. Discovery of novel cross-protective Rickettsia prowazekii T-cell antigens using a combined reverse vaccinology and in vivo screening approach. Vaccine 32, 4968-4976 (2014).

59. Mehla, K. \& Ramana, J. Identification of epitope-based peptide vaccine candidates against enterotoxigenic Escherichia coli: A comparative genomics and immunoinformatics approach. Mol. Biosyst. 12, 890-901 (2016).

60. Delfani, S. et al. In silico analysis for identifying potential vaccine candidates against Staphylococcus aureus . Clin. Exp. Vaccine Res. 4, 99 (2015).

61. Patra, P., Mondal, N., Patra, B. C. \& Bhattacharya, M. Epitope-Based Vaccine Designing of Nocardia asteroides Targeting the Virulence Factor Mce-Family Protein by Immunoinformatics Approach. Int. J. Pept. Res. Ther. 26, 1165-1176 (2020).

62. Totura, A. L. et al. Toll-like receptor 3 signaling via TRIF contributes to a protective innate immune response to severe acute respiratory syndrome coronavirus infection. MBio 6, 1-14 (2015).

\section{Tables}

Table 1. Predicted physicochemical properties of the vaccine construct. 

$(\mathrm{g} / \mathrm{mol})$

Theoretical pI

9.79

10.16

Extinction coefficient

46800

42670

$\left(\mathrm{M}^{-1} \mathrm{~cm}^{-1}\right)$

Estimated half-life

30 hours (mammalian reticulocytes, in vitro).

$>20$ hours (yeast, in

vivo).

$>10$ hours (Escherichia coli, in vivo)

Instability index

Aliphatic index

Grand average of

hydropathicity

(GRAVY)

Estimated solubility
20.26 (stable)

77.84

$-0.273$ 


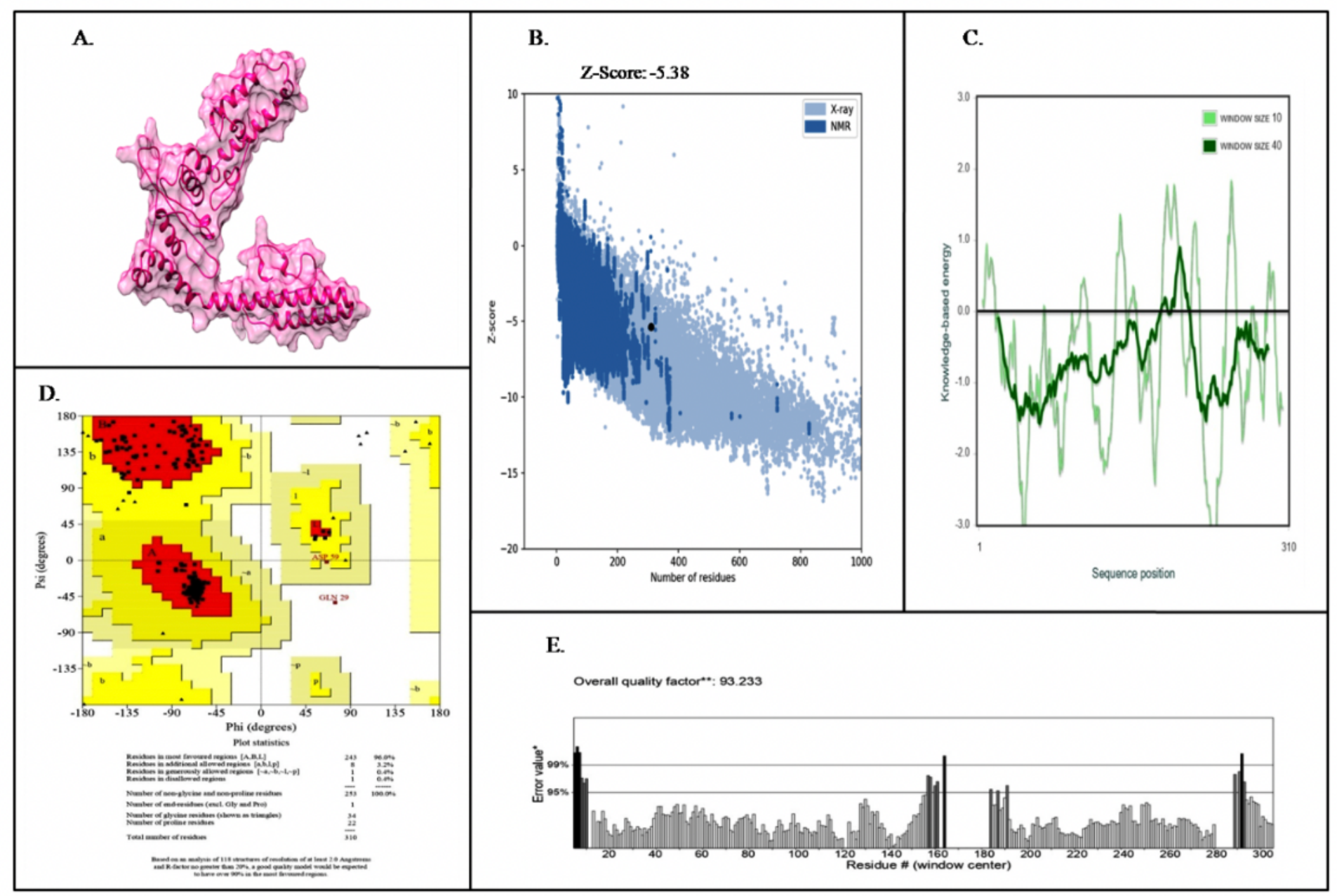

Figure 1

Structural validation of the refined modelled vaccine construct. (A.) Refined tertiary structure of the vaccine protein. (B.) ProSA Z-score (Overall model quality). (C.) ProSA graphical plot (Local model quality). (D.) Ramachandran plot produced using PROCHECK. The zones displayed using different colors viz. red, yellow and light yellow depicts the most favoured regions (96\%), additional allowed regions (3.2\%), and disallowed regions $(0.4 \%)$ respectively. (E.) The ERRAT plot. 


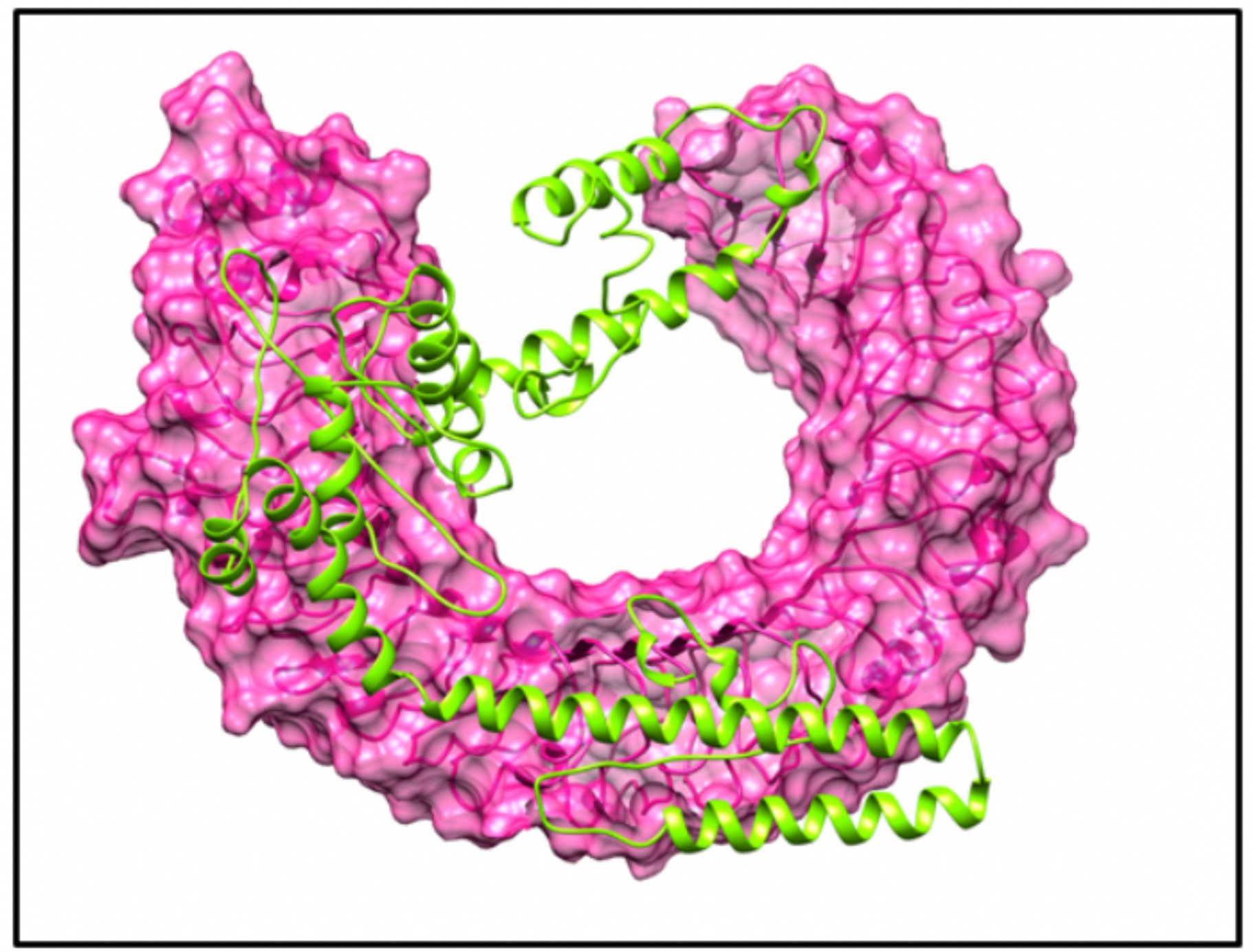

Figure 2

Molecular interaction study of vaccine candidate with immune receptor TLR3 using High Ambiguity Driven (HADDOCK) server. 


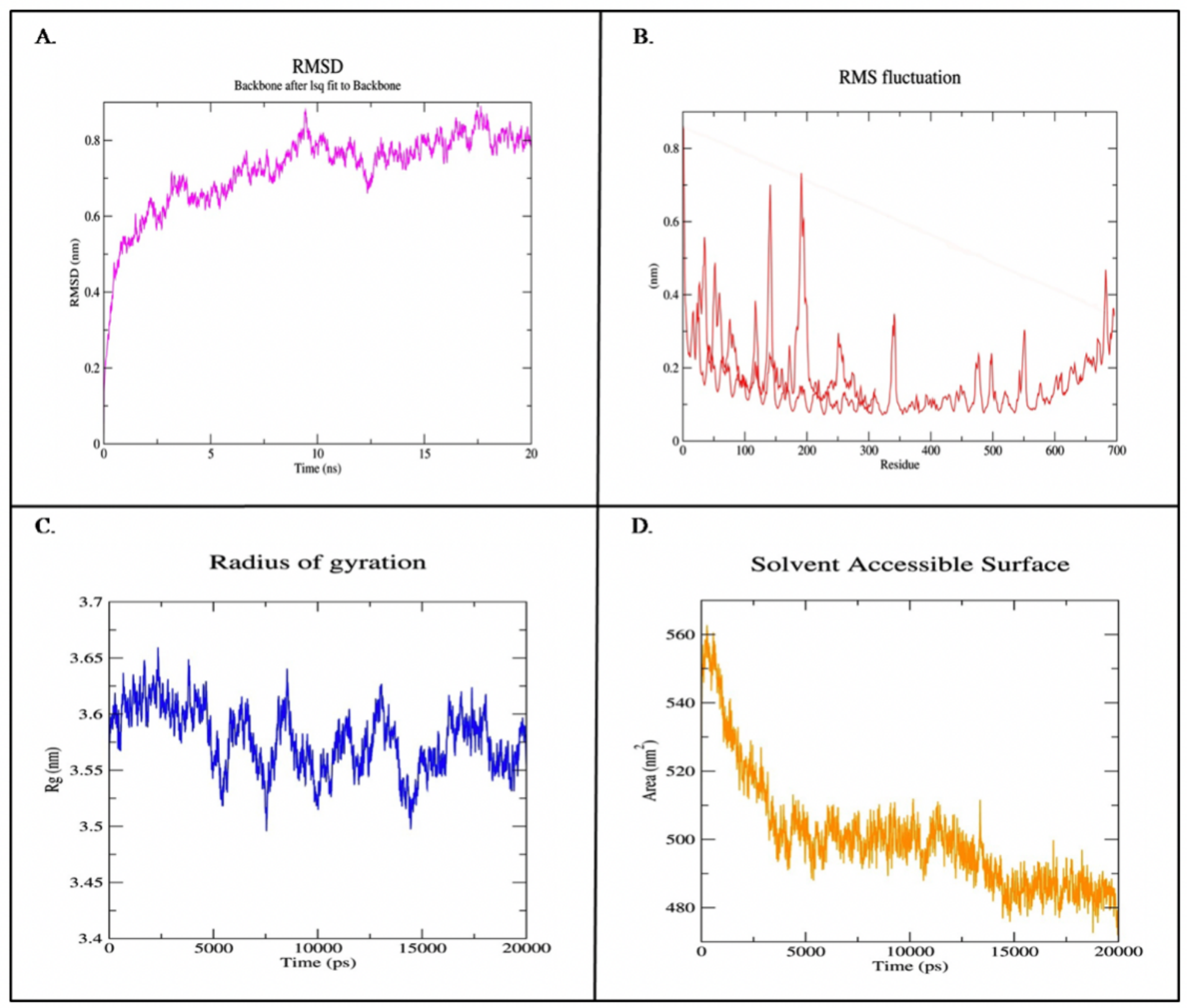

Figure 3

The stability of TLR-3-vaccine candidate as predicted by molecular dynamics simulations. (A.) Root mean square deviation (RMSD) profile. (B.) Root mean square fluctuation (RMSF) profile. (C.) Radius of gyration (Rg) profile, and (D.) Solvent accessible surface area (SASA) profile. The comprehensive computational strategy was utilized to attain insights towards the designed vaccine candidate antigenicity against TLR-3. 


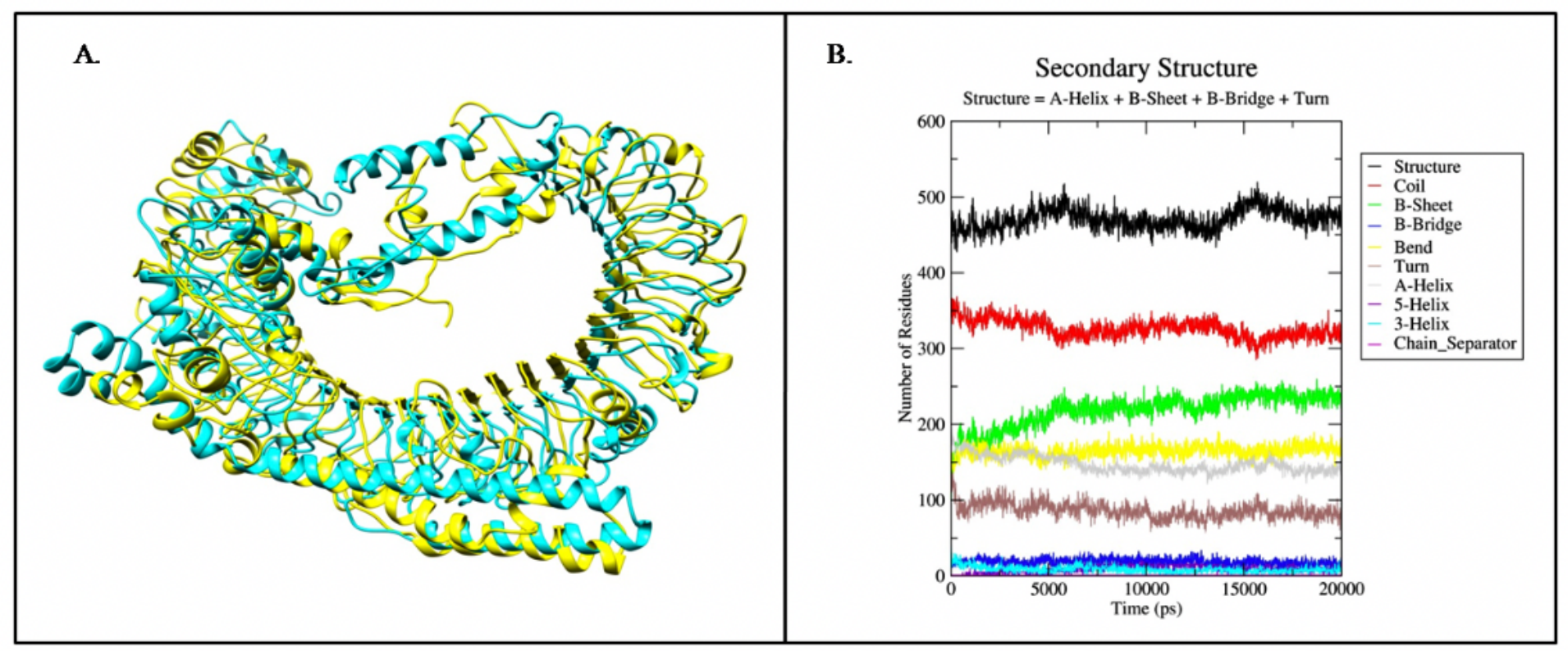

Figure 4

(A.) Superimposed structure for the docked complex of TLR-3-designed vaccine candidate before simulation (cyan color) and after simulation (yellow color), the simulated structure attained compact conformation due to the strong interaction of vaccine candidate into the binding pocket of TLR-3 receptor. (B.) The secondary structure analysis of the stable trajectory for TLR-3 receptor-vaccine candidate was performed by DSSP tool of GROMACS. Maximum of coil conformation is attained (red color) and $\beta$-sheets (green). 


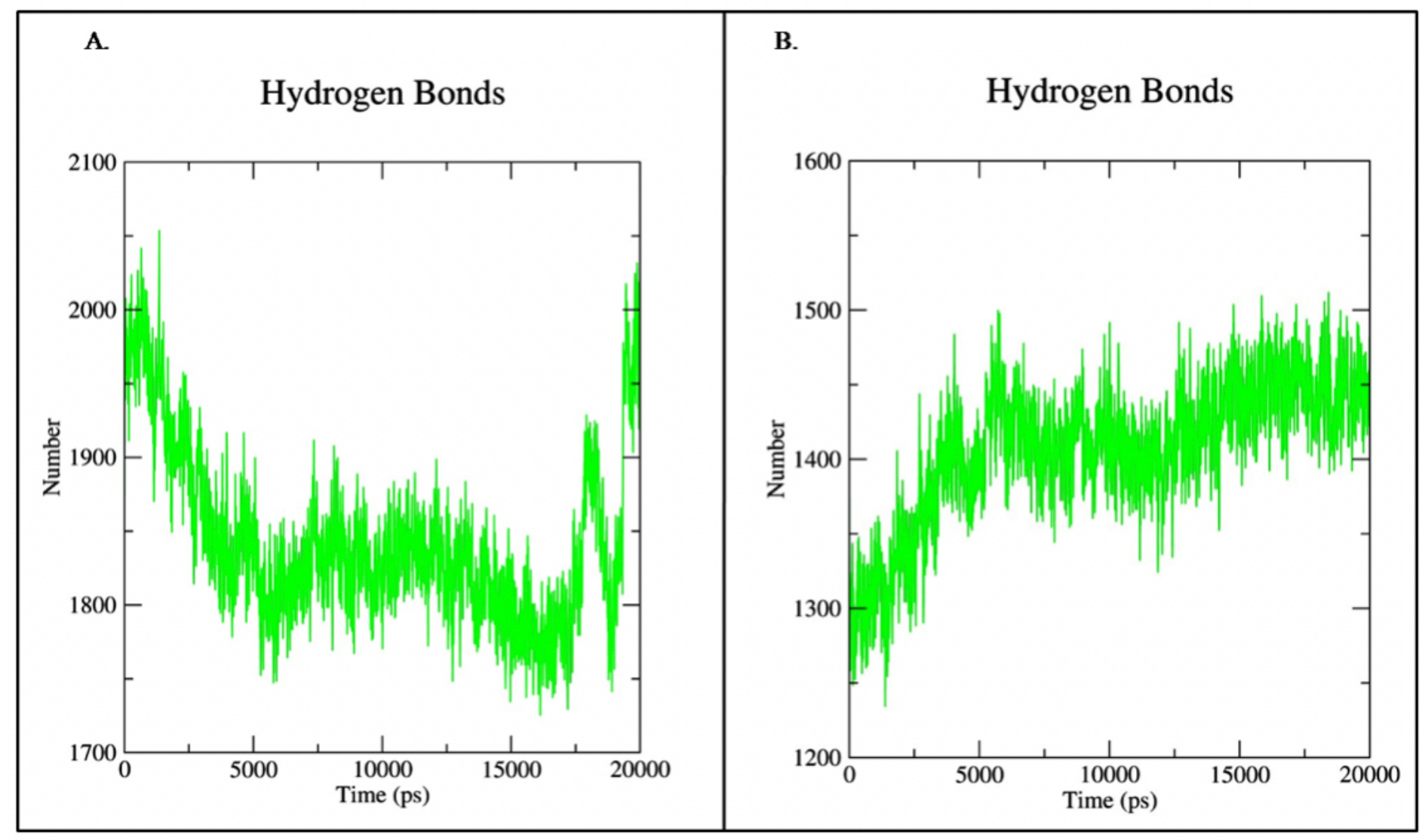

Figure 5

(A.) The statics of consistent high number of hydrogen bonds involved in strong binding of designed vaccine candidate to TLR-3 receptor (B.) Depiction of TLR-3 receptor-vaccine candidate stabilized hydrogen bond with the surrounding solvent throughout the simulation run. 
A.

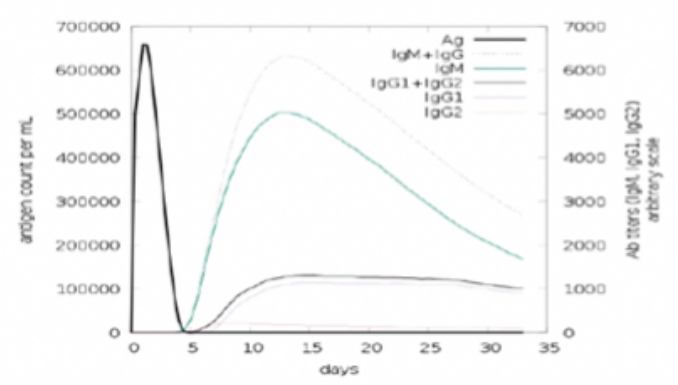

C.

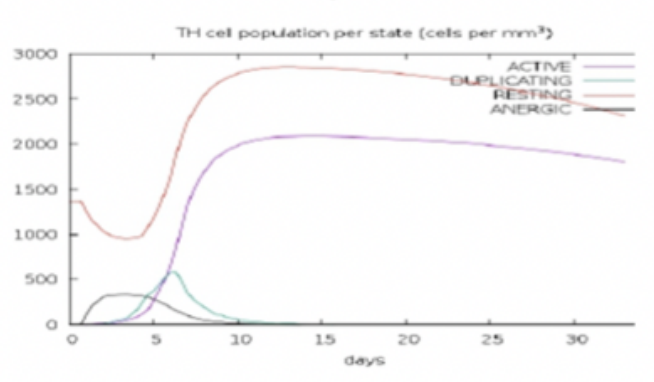

B.

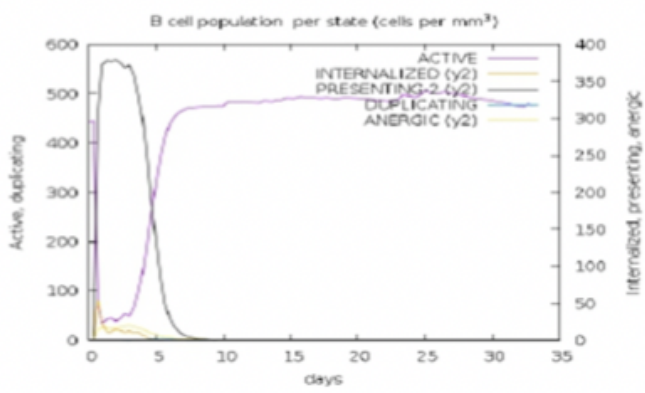

E.

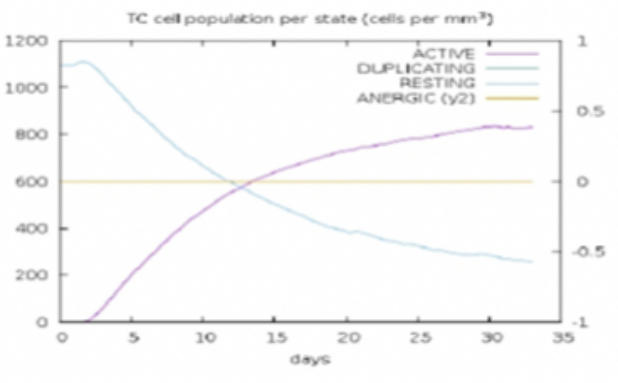

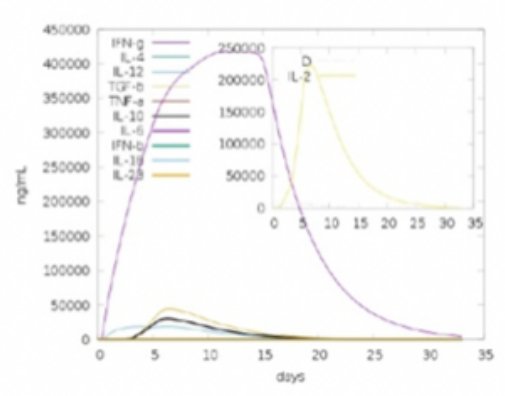

Figure 6

In silico immune simulation results of the vaccine construct using C-ImmSim. (A.) Immunoglobulin generation response on antigen injection. Various sub-classes of immunoglobulin are depicted as colored peaks. (B.) Active B-cell population observed after administrating the vaccine construct. (C.) The demonstration of the emergence of Helper-T cells. (D.) The illustration of the production of cyototoxic-T cells on vaccine administration. In the graphs, RESTING implies to the cells, which were not shown to the antigens while ANERGIC indicates the tolerance level of antigen. (E) Graph displaying the induced cytokine level after vaccine administration. The inset graph illustrates the Simpson Index, D of IL-2. Simpson Index, D was interpreted as the measurement of diversity. 


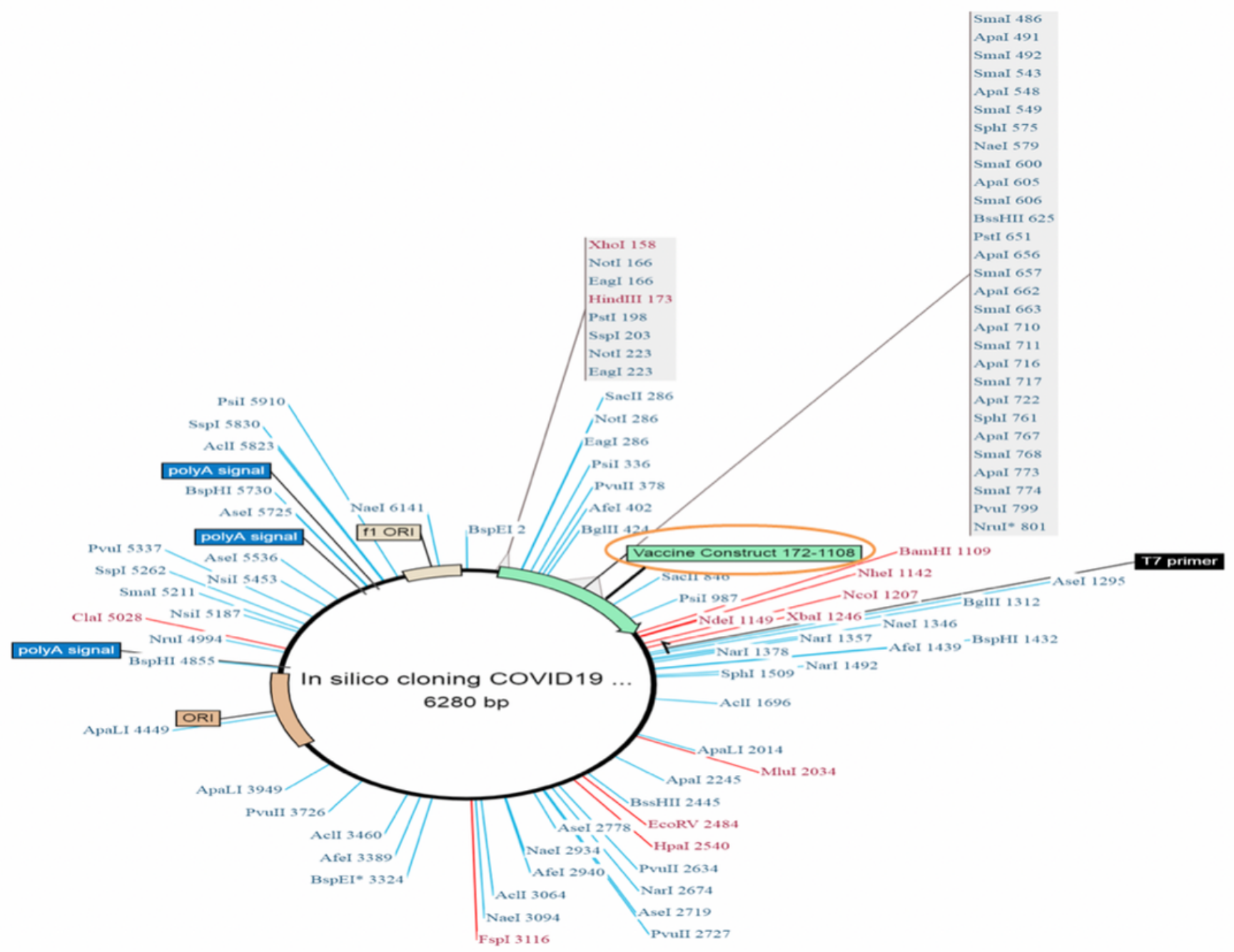

Figure 7

In silico cloning of vaccine construct within the pET28a (+) expression vector.

\section{Supplementary Files}

This is a list of supplementary files associated with this preprint. Click to download.

- Supplementary17.6.20d.docx 